\title{
Difference between penetrating versus blunt ocular trauma in diagnosis, treatment and complications
}

\author{
Ahmed Thabit Alnahdi ${ }^{*}$, Ahmed A. Alaamri², Abdullah K. Alajmi ${ }^{3}$, Malak K. Alkuwaykibi ${ }^{4}$, \\ Ruba A. Bati ${ }^{5}$, Abdulaziz A. Alrabee ${ }^{6}$, Fawaz M. Salman ${ }^{7}$, Sahar Y. AlHawsawi ${ }^{8}$, \\ Ridha S. Aldrees ${ }^{9}$, Abdullah N. Shablahah ${ }^{2}$, Hussain M. Alhasani ${ }^{10}$
}

\author{
${ }^{1}$ Department of Ophthalmology, East Jeddah Hospital, Jeddah, Saudi Arabia \\ ${ }^{2}$ College of Medicine, King Khalid University, Abha, Saudi Arabia \\ ${ }^{3}$ Department of Ophthalmology, Al-Bahar Ophthalmology Center, Al-Sabah, Kuwait \\ ${ }^{4}$ Department of Ophthalmology, King Abdulaziz Specialist Hospital, Sakaka Aljouf, Saudi Arabia \\ ${ }^{5}$ College of Medicine, Umm Al-Qura University, Mecca, Saudi Arabia \\ ${ }^{6}$ Department of Ophthalmology, Ohud Hospital, Medina, Saudi Arabia \\ ${ }^{7}$ College of Medicine, Jordan University of Science and Technology, Irbid, Jordan \\ ${ }^{8}$ Department of Ophthalmology, Hera General Hospital, Mecca, Saudi Arabia \\ ${ }^{9}$ Department of Emergency Medicine, King Fahad Hospital, Al-Ahsa, Saudi Arabia \\ ${ }^{10}$ Department of Ophthalmology, South Al-Qunfudah Hospital, Mecca, Saudi Arabia
}

Received: 24 October 2021

Accepted: 09 November 2021

\section{*Correspondence:}

Dr. Ahmed Thabit Alnahdi,

E-mail: dr_thabitt@hotmail.de

Copyright: (C) the author(s), publisher and licensee Medip Academy. This is an open-access article distributed under the terms of the Creative Commons Attribution Non-Commercial License, which permits unrestricted non-commercial use, distribution, and reproduction in any medium, provided the original work is properly cited.

\begin{abstract}
Ocular trauma can be broadly classified into open and closed injuries. However, many subclassifications were reported based on the causative object and extent of the injury. The diagnosis and management of the various ocular traumas are different based on the underlying etiology, the severity of the case and the potentially related complications. Accordingly, in the present study, we aimed to conduct a thorough discussion about the diagnosis, treatment, complications of the blunt and penetrating ocular traumas and the potential differences between the two types of injuries. Our findings indicated that the diagnosis of the conditions mainly differed in the step of obtaining an adequate history from the affected patients based on the diagnosis of the condition that can be established. Furthermore, a routine examination should also be performed for both cases to enhance the prognosis and preserve vision. In addition, prognosis is usually worse in cases of penetrating or perforating injuries where eye inoculation might be indicated in severe cases. A detailed description of this information is provided within the manuscript based on the etiology of the underlying ocular injury and the related prognostic outcomes.
\end{abstract}

Keywords: Ocular injury, trauma, Management, Treatment, Diagnosis, Complications, Penetrating, Perforating, Blunt, Rupture

\section{INTRODUCTION}

Evidence indicated that the different eye structures are usually exposed to being injured and the mechanism and cause of the injury significantly determine the site and extension of the affected structures. ${ }^{1}$ Additionally, direct trauma usually affects structures within the anterior segment of the eye including the conjunctiva, cornea, trabecular meshwork, crystalline lens, iris and the anterior chamber. The optic nerve, choroid and retina are all structures within the posterior segment and are less likely to be affected by a direct trauma than the structures of the 
anterior segment. ${ }^{1}$ Combined anterior and posterior injury has been associated with the worst prognostic outcomes and vision loss is usually reported in these situations. ${ }^{2-5}$

Ocular trauma can be broadly classified into open and closed injuries. However, many subclassifications were reported based on the causative object and extent of the injury. The diagnosis and management of the various ocular traumas are different based on the underlying etiology, the severity of the case and the potentially related complications. ${ }^{6,7}$ Accordingly, in the present study, we aimed to conduct a thorough discussion about the diagnosis, treatment, complications of the blunt and penetrating ocular traumas and the potential differences between the two types of injuries.

\section{Methods}

This literature review was based on an extensive literature search in Medline, Cochrane and EMBASE databases which was performed on 25 September 2021 using the medical subject headings $(\mathrm{MeSH})$ or a combination of all possible related terms, according to the database. To avoid missing potential studies, a further manual search for papers was done through Google Scholar while the reference lists of the initially included papers. Papers discussing the blunt and penetrating ocular traumas were screened for useful information. No limitations were posed on date, language, age of participants or publication type.

\section{DISCUSSION}

\section{Blunt ocular trauma}

In cases when direct eye trauma had been indicated, the attending physician should adequately examine the eye for a potential injury to the globe. Such injuries should also be suspected in cases of vision defects and continuing eye pain. Furthermore, it was essential to document the timing and mechanism of the injury, which can help with the diagnosis and prognosis of the condition. Obtaining a full medication history from the patient was also essential in these situations. For example, having a previous history of administration of anticoagulants. Following this, adequate physical examination of the injured globe should be initiated. The attending physician should exert serious efforts to look for frank or reduced vision loss, teardrop pupil, globular contour irregularity, shallow anterior chamber and/or hyperemia using the slit-lamp examination. Additionally, in cases of the ruptured globe, the Siedel sign was positive. This can be done using a fluorescence stain and by which the aqueous humor from the injured eye flowed away through the site of injury. ${ }^{8}$ However, it should be noted that looking for this sign was not recommended in cases when a confirmed diagnosis of the ruptured globe had been established. In another context, patients who have severe eye pain and proptosis. A retrobulbar hematoma was also usually associated with these manifestations. Furthermore, vision loss also manifested in cases when globe rupture occured. According to the mechanism of injury, other manifestations might also be associated with blunt trauma to the eye (Figure 1).

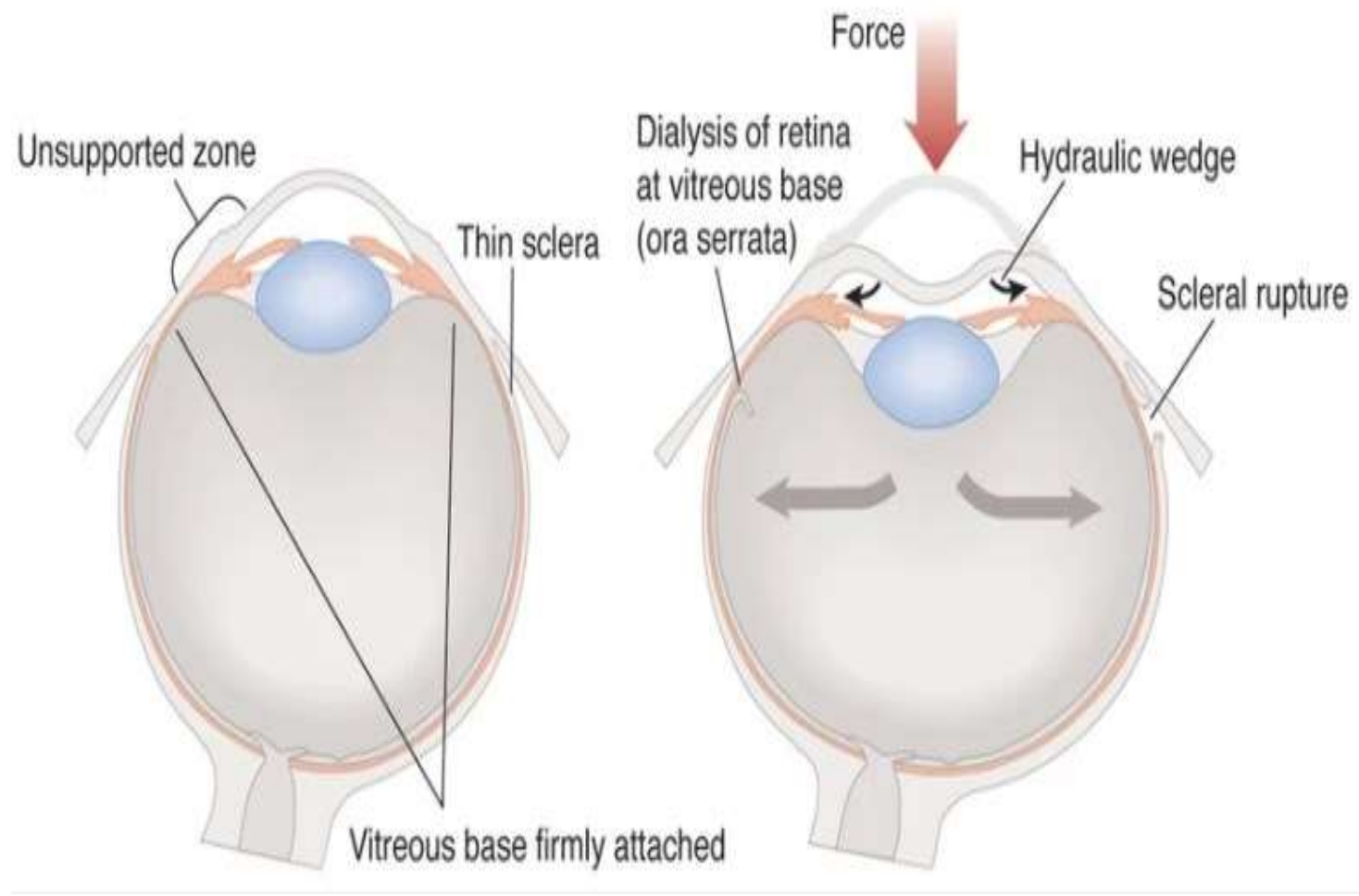

Figure 1: Pathology of blunt ocular trauma. 
These include ecchymosis, periorbital swelling and/or subconjunctival hemorrhage. Following the initial evaluation, examination of the visual acuity in both eyes should be the next step that should also be carefully approached with no manipulation of any underlying injury. It was also recommended to document any underlying pupillary defects. Looking for other facial and intracranial injuries also helped with the diagnosis of the condition. Associated orbital fractures might be indicated in cases of multiple hematomas and globe ruptures. ${ }^{9}$ To evaluate the presence of retrobulbar hematoma, intrinsic globe deformity, entrapment, extra-ocular motility evaluation should also be considered. Conducting relevant laboratory investigations might also be needed in some cases based on the clinical and personal history of the presenting patients. Requesting computed tomography might also be necessary in cases when other maxillofacial injuries were suspected. Also, conducting imaging modalities can furtherly detect the underlying foreign body, vitreous hemorrhage and scleral disruption. ${ }^{10}$

In cases when globe rupture had been indicated, patients should be managed at the emergency department to provide the necessary management to enhance the prognosis of the condition and to prevent any further extrusion of the intraocular contents. It was also recommended that these patients should administer antiemetics to prevent the patient from vomiting. ${ }^{11}$ This was usually done to reduce the potential to increase the intraocular pressure that might lead to increased loss of the aqueous fluid through the injury. Analgesia should be provided as required by the patient and manipulation of the injury and eye contents should be avoided. Placing a rigid eye shield and making the patient in a semi-setting position should also be considered. ${ }^{12-14}$ In addition, antiemetics and analgesics should also be provided in cases of retrobulbar hematoma. Inferior cantholysis and lateral canthotomy should be definitely done in these patients to achieve immediate decompression. This should be done within the emergency department by either the attending emergency physician or ophthalmologist. Additionally, the duration of conducting this approach was also critical and can determine the prognosis of vision in the affected patients as evidence indicates that vision loss was usually established after 4 hours since the symptoms were reported. It was also recommended that the affected patients should administer prophylactic antibiotics to prevent the development of secondary infections (as endophthalmitis). Surgical repair should also be emergently done by an ophthalmologist in cases of retrobulbar hematoma, and globe injury after adequate evaluation by the attending ophthalmologist.

Evidence indicated the variance of complications that can follow blunt ocular trauma were stratified based on the extent, location, depth and mechanism of injury. For instance, superficial injuries can only lead to corneal scarring. On the other hand, deep injuries can lead to post-traumatic glaucoma, choroidal rupture, endophthalmitis, corneal rupture and cataracts. Variable intensities of vision can also develop in the affected eye. The sequel of the blunt ocular trauma was a significant predictor for the development of ocular complications and visual prognosis. For instance, monocular blindness in one quarter was documented in a patient that suffered from ocular trauma. ${ }^{15} \mathrm{~A}$ previous investigation also demonstrated that the visual acuity was usually better among patients that suffered from blunt ocular trauma than the penetrating one. On the other hand, the same authors also concluded that glaucoma was more frequent in patients with blunt ocular traumas. ${ }^{16}$ Evidence also indicated that the worst visual-related prognosis can occur in cases with blunt trauma where a ruptured globe was indicated. Furthermore, the absence of red reflex, afferent pupillary defects, eyelid laceration and initially worsened visual acuity were also other factors that can significantly predict a poor prognosis in the affected patients with blunt ocular trauma. ${ }^{17}$ In cases of sclopetaria retinae or commotio retinae that can develop secondary to blunt ocular trauma, a previous investigation reported that around $25 \%$ of their included population had worsened visual acuity in the affected eyes. ${ }^{18}$ Retinal detachment, hyperemia and vitreous hemorrhage were signs that can predict the presence of a worsened prognosis following blunt ocular trauma. ${ }^{19}$ In their case series, Wang et al also demonstrated that a worse prognosis of visual acuity was also associated with the occurrence of traumatic optic neuropathy. ${ }^{20,21}$ Finally, it should be noted that the mechanism and extent of the primary lesion were the main determinants of the severity of the case and the anticipated prognosis. Evidence indicated that the ocular trauma score can be effectively used to predict and evaluate the functional outcomes of the traumatized eye and predict the prognosis. ${ }^{21}$ Inoculation might be the only management modality that can be offered to the affected patients in cases when the surgical repair cannot adequately manage the underlying severe trauma.

\section{Penetrating ocular trauma}

Trauma by a sharp object can be penetrating or perforating which can be differentiated from each other by the full thickness end laceration, which was single with the penetrating trauma and multiple with the perforating one and the presence of exit wound, which was present with the perforating trauma only. ${ }^{22,23}$ Furthermore, prolapse of the internal eye contents might also be associated with these situations. ${ }^{24}$ The momentum of the object and the site where the trauma took place were the two factors that can significantly impact the severity of the underlying situation. ${ }^{25,26}$ Domestic accidents, assaults and sports can all lead to the development of penetrating trauma. ${ }^{25-28}$ In these situations, the eye was impacted by sharp objects such as needles or nails or by high-velocity missiles as small pieces of metals (Figure 2). 


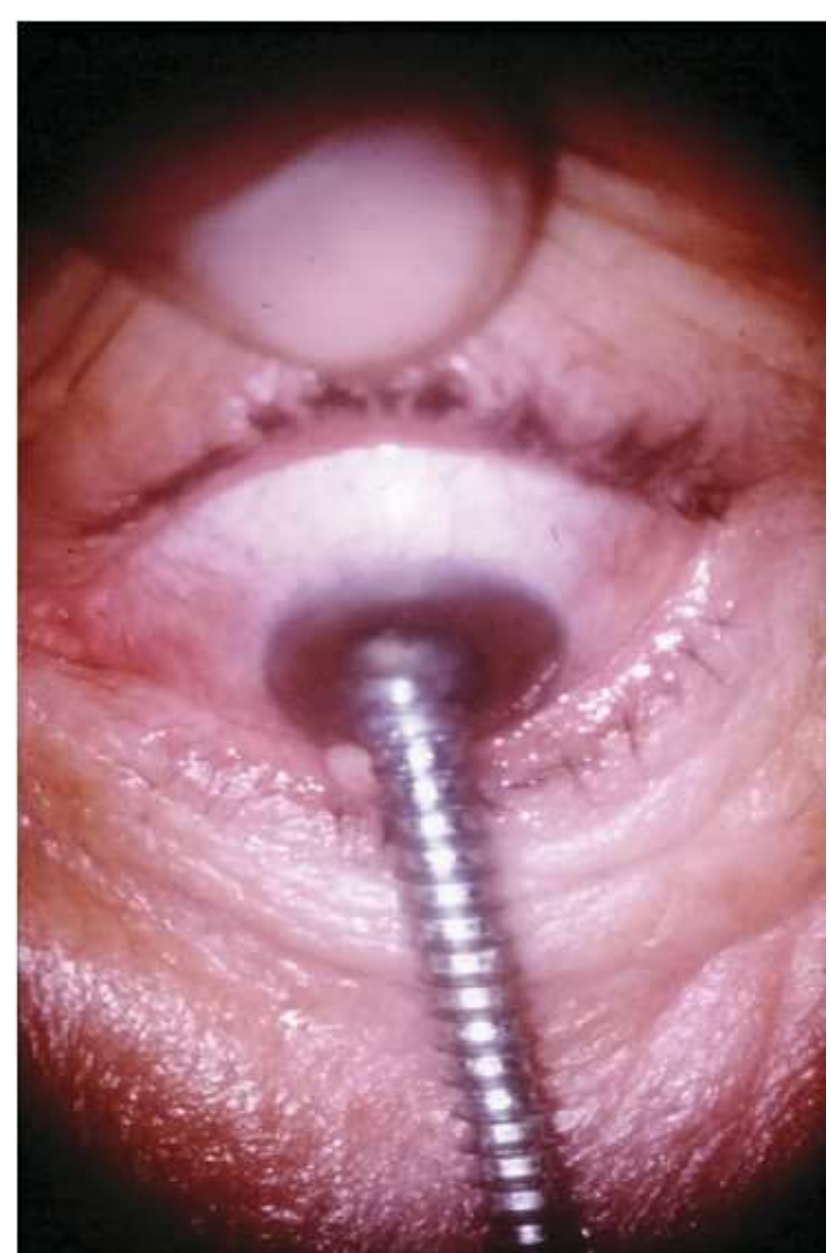

Figure 2: An example of a penetrating ocular injury by a screw. ${ }^{26}$

The size, speed and composition of the causative substance were the main factors that can determine the extent and severity of the trauma and injury to the globe. ${ }^{29}$ In these situations also, the associated lacerations were usually well defined as a result of the sharp edges of the penetrating object. On the other hand, the kinetic energy of a flying object also determined the extent of the injury in some cases. ${ }^{25,27}$ When the cornea was the only affected structure, the impaction of the structures within the anterior segment was less frequent. Therefore, vision affection was not likely to occur in these situations. On the other hand, when deeper injuries were usually observed, the scarring might be associated secondary to the prolonged healing process. Consequently, the vision of the affected patients might be significantly impacted. ${ }^{25}$ Trauma within the anterior segment that involved the anterior lens capsule usually led to the development of diffuse or localized lenticular opacity. Scarring and vitreoretinal traction might also be observed in some cases that suffered from posterior wounds. In this context, these events can significantly lead to the development of complex retinal detachment. ${ }^{25,27}$ Surgical management of these situations was urgently required. It was usually done to maintain and control the intraocular pressure within normal range, restore the normal anatomical structure of the affected eye and reduce the risk of developing secondary infections and related complications..$^{25,30,31}$ Eye enucleation might also be surgically indicated in certain situations such as vitreous abscess, infections, tractional retinal detachment, cataract and anterior synechiae. During the follow up period, further management indications can be furtherly required in some situations.

Obtaining an adequate history from the affected patient was a critical step to diagnose a penetrating trauma adequately. Accordingly, plan to carefully manage and monitor the case during follow up to enhance the visual prognosis. ${ }^{32}$ Restoring of preserving vision, relieving pain and achieving good esthetic outcomes were the main goals of the management approaches. In cases when perforation was suspected, conducting motility testing, lids manipulation and measurement of the intraocular pressure should not be done in these situations. Gently covering the eye should then be done in these situations and was approached by a protective shield, eye pad or sterile gauze. ${ }^{25}$ Cranial imaging should also be conducted to determine the extent of the injury and make sure no remaining foreign bodies have been avoided. ${ }^{33}$ Furthermore, prophylactic administration of antibiotics should be indicated. It had been furtherly demonstrated that uveitis can also affect the non-injured eye. This occured as a result of the potential spread of uveal pigments into the blood, which altered an antibody reaction and immune response and potentially leading to an autoimmune inflammatory reaction to the non-injured eye $^{34,35}$ Eye inoculation of the injured eye should be indicated in cases when inflammation of the other healthy eye developed and in cases when vision in this eye was of a poor prognostic value. Reduced cooperation can be a major issue when managing these cases in children, which can affect the diagnosis process and treatment compliance. Then, other management approaches were similar among adults and children. ${ }^{36}$ The presence of amblyopia can also affect the prognosis. The presence of complications in these situations was significantly determined by the severity and extent of the injury. Although many advances have been reported with the microsurgical techniques for managing these situations, evidence still indicated that the prognosis was still poor and mostly depended on the severity of the cases and the extent of the injury. ${ }^{34,37-39}$

\section{CONCLUSION}

Our findings indicate that the diagnosis of the conditions mainly differs in the step of obtaining an adequate history from the affected patients based on the diagnosis of the condition that can be established. A routine examination should also be performed for both cases to enhance the prognosis and preserve vision. Prognosis is usually worse in cases of penetrating or perforating injuries, where eye inoculation might be indicated in severe cases. 
Funding: No funding sources

Conflict of interest: None declared

Ethical approval: Not required

\section{REFERENCES}

1. Canavan YM, Archer DB. Anterior segment consequences of blunt ocular injury. $\mathrm{Br} \quad \mathrm{J}$ Ophthalmol. 1982;66(9):549-55.

2. Eagling EM. Perforating injuries of the eye. $\mathrm{Br} \mathbf{J}$ Ophthalmol. 1976;60(11):732-6.

3. Bauza AM, Emami P, Soni N, Holland BK, Langer $\mathrm{P}$, Zarbin $\mathrm{M}$, et al. A 10-year review of assaultrelated open-globe injuries at an urban hospital. Graefes Arch Clin Exp Ophthalmol. 2013;251(3):653-9.

4. Gyasi M, Amoaku W, Adjuik M. Epidemiology of hospitalized ocular injuries in the upper East region of ghana. Ghana Med J. 2007;41(4):171-5.

5. Ramirez DA, Porco TC, Lietman TM, Keenan JD. Ocular injury in United States emergency departments: seasonality and annual trends estimated from a nationally representative dataset. Am J Ophthalmol. 2018;191:149-55.

6. Sukati V. Ocular injuries-a review. Afr Vision Eye Health. 2012;71(2):86-94.

7. MacEwen CJ. Ocular injuries. J Royal Coll Surgeon Edinburgh. 1999;44(5):317-23.

8. Desai P, MacEwen CJ, Baines P, Minassian DC. Incidence of cases of ocular trauma admitted to hospital and incidence of blinding outcome. $\mathrm{Br} \mathrm{J}$ Ophthalmol. 1996;80(7):592-6.

9. Thomas R, McManus JG, Johnson A, Mayer P, Wade C, Holcomb JB. Ocular injury reduction from ocular protection use in current combat operations. J Trauma. 2009;66(4):99-103.

10. Mohseni M, Blair K, Bragg BN. Blunt eye trauma. Treasure Island (FL): StatPearls Publishing; 2021.

11. Mwangi N, Mutie DM. Emergency management: penetrating eye injuries and intraocular foreign bodies. Commun Eye Health. 2018;31(103):70-1.

12. Nemet AY, Asalee L, Lang Y, Briscoe D, Assia EI. Ocular paintball injuries. Israel Med Assoc J. 2016;18(1):27-31.

13. Hoskin AK, Yardley AM, Hanman K, Lam G, Mackey DA. Sports-related eye and adnexal injuries in the Western Australian paediatric population. Acta Ophthalmologica. 2016;94(6):407-10.

14. Gelston CD. Common eye emergencies. Am Fam Physician. 2013;88(8):515-9.

15. Zungu T, Mdala S, Manda C, Twabi HS, Kayange P. Characteristics and visual outcome of ocular trauma patients at Queen Elizabeth central hospital in Malawi. PloS One. 2021;16(3):0246155.

16. Ram J, Verma N, Gupta N, Chaudhary M. Effect of penetrating and blunt ocular trauma on the outcome of traumatic cataract in children in northern India. J Trauma Acute Care Surg. 2012;73(3):726-30.
17. Rahman I, Maino A, Devadason D, Leatherbarrow B. Open globe injuries: factors predictive of poor outcome. Eye. 2006;20(12):1336-41.

18. Blanch RJ, Good PA, Shah P, Bishop JR, Logan A, Scott RA. Visual outcomes after blunt ocular trauma. Ophthalmology. 2013;120(8):1588-91.

19. Yucel OE, Demir S, Niyaz L, Sayin O, Gul A, Ariturk N. Clinical characteristics and prognostic factors of scleral rupture due to blunt ocular trauma. Eye. 2016;30(12):1606-13.

20. Wang BH, Robertson BC, Girotto JA, Liem A, Miller NR, Liff $\mathrm{N}$, et al. Traumatic optic neuropathy: a review of 61 patients. Plast Reconstr Surg. 2001;107(7):1655-64.

21. Kuhn F, Maisiak R, Mann L, Mester V, Morris R, Witherspoon CD. The ocular trauma score (OTS). Ophthalmol Clinic North Am. 2002;15(2):163-5.

22. Kuhn F, Morris R, Witherspoon CD, Heimann K, Jeffers JB, Treister G. A standardized classification of ocular trauma. Ophthalmology. 1996;103(2):2403.

23. Katz SE. Ocular trauma: principles and practice. LWW; 2003.

24. Smith D, Wrenn K, Stack LB. The epidemiology and diagnosis of penetrating eye injuries. Acad Emerg Med. 2002;9(3):209-13.

25. McGwin G, Xie A, Owsley C. Rate of eye injury in the United States. Arch Ophthalmol. 2005;123(7):970-6.

26. Scott R. The injured eye. Philo Transaction Royal Soc Biolog Sci. 2011;366:251-60.

27. Potockova A, Strmen P, Krasnik V, Olah Z. Mechanical injuries of the eye. Bratislavske Lekarske Listy. 2010;111(6):329-35.

28. Napier SM, Baker RS, Sanford DG, Easterbrook M. Eye injuries in athletics and recreation. Surv Ophthalmol. 1996;41(3):229-44.

29. Huang Z, Chen L, Zeng Y, Lin C. Clinical features of perforating eye injuries complicated with intraocular foreign bodies located at the posterior global wall. Eye Sci. 2013;28(4):180-4.

30. Lee DE, Ryoo HW, Moon S, Ahn JY, Kim JH, Kim JY. Epidemiology and risk factors for sports- and recreation-related eye injury: a multicenter prospective observational study. Int J Ophthalmol. 2021;14(1):133-40.

31. Weichel ED, Bower KS, Colyer MH. Chorioretinectomy for perforating or severe intraocular foreign body injuries. Graefe's Arch Clinic Experiment Ophthalmol. 2010;248(3):31930.

32. Cinotti AA, Maltzman BA. Prognosis and treatment of perforating ocular injuries. John Luhr memorial lecture. Ophthalm Surg. 1975;6(4):54-61.

33. Cass SP. Ocular injuries in sports. Curr Sport Med Reps. 2012;11(1):11-5.

34. Thompson CG, Kumar N, Billson FA, Martin F. The aetiology of perforating ocular injuries in children. Br J Ophthalmol. 2002;86(8):920-2. 
35. Beby F, Kodjikian L, Roche O, Donate D, Kouassi $\mathrm{N}$, Burillon $\mathrm{C}$, et al. Perforating ocular injuries in children: a retrospective study of 57 cases. J Fr Ophtalmol. 2006;29(1):20-3.

36. Apoorva MN, Hamsaveni V. Ocular injuries-a review article. JAIMS. 2018;3(5).

37. Cleary PE, Ryan SJ. Experimental posterior penetrating eye injury in the rabbit. I. Method of production and natural history. $\mathrm{Br} \mathrm{J}$ Ophthalmol. 1979;63(5):306-11.

38. Mackiewicz J, Machowicz-Matejko E, Sałaga-Pylak M, Piecyk-Sidor M, Zagórski Z. Work-related, penetrating eye injuries in rural environments. Ann Agri Environment Med. 2005;12(1):27-9.

39. Smith D, Wrenn K, Stack LB. The epidemiology and diagnosis of penetrating eye injuries. Acad Emerg Med. 2002;9(3):209-13.

Cite this article as: Alnahdi AT, Alaamri AA, Alajmi AK, Alkuwaykibi MK, Bati RA, Alrabee AA, et al. Difference between penetrating versus blunt ocular trauma in diagnosis, treatment and complications. Int J Community Med Public Health 2021;8:6101-6. 